\title{
Implantologie chez les patients bénéficiant ou ayant bénéficié d'une chimiothérapie et/ou d'une radiothérapie anticancéreuse
}

\author{
Margainaud JP \\ Institut Gustave Roussy, Villejuif, France
}

Les complications de la chimiothérapie anticancéreuse classique sont en principe réversibles: il est donc possible, à la fin du traitement, lorsque le patient est en rémission, d'envisager la pose d'implant sous réserve d'un contrôle de sa formule sanguine. Par contre, les bisphosphonates à visée anticancéreuse contre-indiquent tout acte implantaire en raison du risque d'ostéonécrose .

Le problème est différent pour la radiothérapie dont les séquelles sont définitives avec un risque croissant d'ostéoradionécrose avec le temps. Il n'y a pas d'évidence scientifique à ce sujet car il ne semble pas exister de séries significatives de patients implantés en territoire irradié avec une dose supérieure à 50 grays. Il faut donc considérer que, comme tout acte chirurgical dans ce cas, la pose d'implant représente un risque à court, moyen et long terme.

C'est ici qu'intervient la notion de bénéfice-risque car l'enjeu est différent s'il s'agit de traiter une édentation simple ou de stabiliser une prothèse maxillo-faciale qui permettra au malade de retrouver une élocution, une alimentation et une esthétique acceptables. Quand cela est possible, il faut éviter l'implantation dans les zones à risques; dans le cas contraire, la décision doit être prise avec le patient qui doit rester demandeur pour ce type de réhabilitation après avoir été prévenu de tous les risques encourus par un consentement éclairé. L'acte chirurgical se fera avec toute s les précautions d'usage pour une intervention sur un os irradié. Si cela est réalisable en fonction du timing thérapeutique, implanter avant la radiothérapie constitue une alternative séduisante: en effet, la logique laisse à penser qu'il est moins risqué d'irradier un implant que d'implanter en territoire irradié.

En conclusion, si l'implantologie chez les patients ayant bénéficié d'une chimiothérapie, hormis pour les bisphosphonates, ne pose pas de problème, il convient en territoire irradié d'agir avec prudence et raison, en fonction du rapport bénéfice-risque après information et consentement du patient.

Correspondance : margaina@igr.fr

This is an Open Access article distributed under the terms of the Creative Commons Attribution-Noncommercial License 3.0, which permits unrestricted use, distribution, and reproduction in any noncommercial medium, provided the original work is properly cited. 